\title{
Unsteady RANS and LES Simulation of an Ideal Rankine Vortex Decay
}

\author{
Md. Shahjahan Ali, ${ }^{1}$ Takashi Hosoda, ${ }^{2}$ and Ichiro Kimura ${ }^{3}$ \\ ${ }^{1}$ Civil Engineering Department, Khulna University of Engineering and Technology, Khulna 9203, Bangladesh \\ ${ }^{2}$ Department of Urban Management, Kyoto University, Kyoto 615-8540, Japan \\ ${ }^{3}$ Faculty and Graduate School of Engineering, Hokkaido University, Sapporo 060-8623, Japan
}

Correspondence should be addressed to Md. Shahjahan Ali, bablu41@yahoo.com

Received 27 July 2011; Revised 7 December 2011; Accepted 3 February 2012

Academic Editor: Ghassan Chehab

Copyright (c) $2012 \mathrm{Md}$. Shahjahan Ali et al. This is an open access article distributed under the Creative Commons Attribution License, which permits unrestricted use, distribution, and reproduction in any medium, provided the original work is properly cited.

The 3D numerical simulation was carried out for an idealized Rankine vortex using nonlinear $k-\varepsilon$ model (one kind of RANS model) and large eddy simulation (LES) techniques. In this 3D simulation, the vortex flow field was given to rotate with the vertical axis in a free surface rectangular domain. In order to investigate the predictability of standard (linear) and non-linear $k-\varepsilon$ models, the decay of a trailing vortex was simulated and compared with previous DNS data. The governing equations for mean velocities and turbulent flows were discretized with the finite volume method based on a staggered grid system. It was observed that in the growth phase as well as in stabilized phase of turbulence, the decay rate of tangential velocity by RANS model was well comparable with LES simulation as well as previous DNS data. However, in the decay phase of turbulence, RANS model showed slightly faster decay of tangential velocity due to its slower decay of turbulence compared to LES or DNS. The patterns as well as magnitudes of secondary currents predicted by RANS and LES models were well comparable to each other.

\section{Introduction}

The basic types of plane vortices can be classified into two categories: one with slower velocity at center and maximum at sides and the other with maximum at center and minimum velocity at edges. The rotary fluid motion of the first one is called the solid body rotation, since it is similar to the fluid motion filled in a rotating hollow box. On the other hand, if a long circular rod rotates in a fluid with constant velocity around its axis, the fluid velocity is found highest and equal to the velocity of rod at the rod's surface (due to adhesion), and with increasing distance from the rod, the velocity is diminished in inverse proportional to the distance. Such a fluid motion is called a potential vortex. Fluid motion composed of a potential vortex and solid body rotation is called Rankine vortex (Figure 1) after the fluid dynamicist Rankine. The radial distance from the center to the maximum tangential velocity is called the radius of vortex core. For a steady circular motion without a velocity component normal to the plane of rotation, the Rankine vortex is the only possible vortex whose velocity is zero at the center as well as far away from it. In addition to these basic vortices, there are other time-dependent rotary motions that have azimuthal velocity component as well as radial and axial components.

The existence of such vortices is not only limited to natural phenomena but also in many engineering applications. The Rankine vortex has been used extensively in various studies: for instance, to model the natural phenomena such as hurricanes and tornados, to study the vortex wake hazard caused by flow obstructions, to predict the decay of wingtip vortices, and so forth. Although some experimental and theoretical studies ([1-3], etc.) as well as DNS simulations [4] were successfully performed to study the structure and decay of such vortex, all of them were performed considering $2 \mathrm{D}$ vortex field.

As long as the flow is laminar, the only problem is to solve a system of discretized equations. The accuracy of results depends on both the grid and discretization scheme. The moment that the flow becomes turbulent, a chaotic, random motion is observable. In this case, the instantaneous flow values at a fixed location in the flow field show a fluctuating behavior. Therefore, in order to study such flows numerically, 


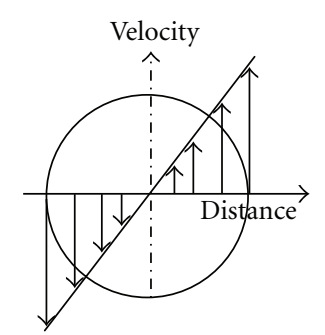

(a)

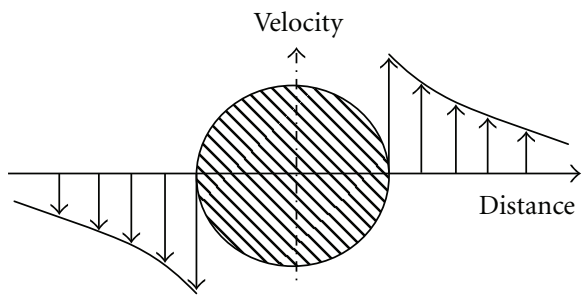

(b)

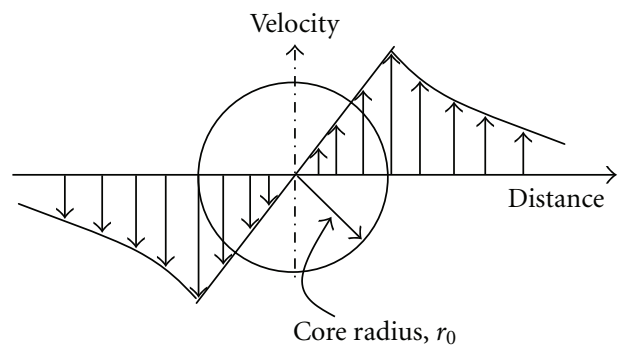

(c)

FIgURE 1: Velocity distribution in (a) solid body rotation, (b) potential vortex, and (c) Rankine vortex.

different approaches have been proposed. They have different accuracies, and sometimes their predictability depends on the type of flows. The major approaches to study turbulent flows numerically are Reynolds-averaged Navier-Stokes (RANS), large eddy simulation (LES), and direct numerical simulation (DNS). In this study, the 3D numerical simulations were carried out using non-linear $k-\varepsilon$ model (one kind of RANS model) and large eddy simulation (LES) techniques. The idea of LES comes from the fact that the small scales show an isotropic behavior regardless of the type of flow and boundary conditions. Hence, if the large scales are resolved, it is accurate enough to model the small scales which are called the subgrid scale or SGS. In order to get reasonable accuracy, the grid should be fine to some extent. Important difference between RANS and LES governing equations is the method of filtering. While in RANS the filtering is performed in time, in LES the filtering is performed in space. Another difference compared to RANS is that, as turbulence is a timedependent three-dimensional phenomenon, the governing equations in LES also need to solve in unsteady threedimensional format. Another fact regarding LES is that the grid is supposed to be fine enough to capture some part of scales in the inertial subrange. These differences make the LES much more expensive than RANS models.
The work presented in this paper can be classified into twofold. In the first part, unsteady numerical simulation with $3 \mathrm{D}$ velocity field was carried out for a turbulent trailing vortex using non-linear $k-\varepsilon$ model. To assess the predictability of non-linear $k-\varepsilon$ model, general features of vortex decay are compared with previous DNS data. The simulation was also performed using standard $k-\varepsilon$ model and comparison is shown with non-linear model. In the second part, the 3D numerical simulations were carried out for an idealized Rankine vortex using non-linear $k-\varepsilon$ model and large eddy simulation (LES). 3D staggered grid was used for both RANS and LES simulations. Comparison is shown for the general flow features such as temporal change of vortex decay, radial distribution of tangential velocity, and water surface profile.

\section{Numerical Model and Simulation Details}

2.1. Nonlinear $k-\varepsilon$ Model. Following 3D flow equations [5-7] were used in unsteady RANS calculation for the chosen flow field.

Continuity equation:

$$
\frac{\partial U_{i}}{\partial x_{i}}=0
$$

Momentum equation:

$\frac{\partial U_{i}}{\partial t}+\frac{\partial U_{j} U_{i}}{\partial x_{j}}=g_{i}-\frac{1}{\rho} \frac{\partial P}{\partial x_{i}}+\frac{\partial}{\partial x_{j}}\left(-\overline{u_{i} u_{j}}\right)+\nu \frac{\partial^{2} U_{i}}{\partial x_{j}^{2}}$

k-equation:

$\frac{\partial k}{\partial t}+\frac{\partial k U_{j}}{\partial x_{j}}=-\overline{u_{i} u_{j}} \frac{\partial U_{i}}{\partial x_{j}}+\frac{\partial}{\partial x_{j}}\left\{\left(\frac{\nu_{t}}{\sigma_{k}}+\nu\right) \frac{\partial k}{\partial x_{j}}\right\}-\varepsilon$.

$\varepsilon$-equation:

$\frac{\partial \varepsilon}{\partial t}+\frac{\partial \varepsilon U_{j}}{\partial x_{j}}=-c_{\varepsilon} \frac{\varepsilon}{k} \overline{u_{i} u_{j}} \frac{\partial U_{i}}{\partial x_{j}}+\frac{\partial}{\partial x_{j}}\left\{\left(\frac{\nu_{t}}{\sigma_{\varepsilon}}+\nu\right) \frac{\partial \varepsilon}{\partial x_{j}}\right\}-c_{\varepsilon 2} \frac{\varepsilon^{2}}{k}$.

Turbulence model (nonlinear):

$$
-\overline{u_{i} u_{j}}=v_{t} S_{i j}-\frac{2}{3} k \delta_{i j}-\frac{k}{\varepsilon} v_{t} \sum_{\beta=1}^{3} c_{\beta}\left(S_{\beta i j}-\frac{1}{3} S_{\beta \alpha \alpha} \delta_{i j}\right) .
$$

Here,

$$
\begin{gathered}
v_{t}=c_{\mu} \frac{k^{2}}{\varepsilon}, \quad S_{i j}=\frac{\partial U_{i}}{\partial x_{j}}+\frac{\partial U_{j}}{\partial x_{i}}, \quad \Omega_{i j}=\frac{\partial U_{i}}{\partial x_{j}}-\frac{\partial U_{j}}{\partial x_{i}}, \\
S=\frac{k}{\varepsilon} \sqrt{\frac{1}{2} S_{i j} S_{i j}}, \quad \Omega=\frac{k}{\varepsilon} \sqrt{\frac{1}{2} \Omega_{i j} \Omega_{i j}} .
\end{gathered}
$$




$$
\begin{gathered}
\text { In (5), } \\
S_{1 i j}=\frac{\partial U_{i}}{\partial x_{\gamma}} \frac{\partial U_{j}}{\partial x_{\gamma}}, \quad S_{2 i j}=\frac{1}{2}\left(\frac{\partial U_{\gamma}}{\partial x_{i}} \frac{\partial U_{j}}{\partial x_{\gamma}}+\frac{\partial U_{\gamma}}{\partial x_{j}} \frac{\partial U_{i}}{\partial x_{\gamma}}\right), \\
S_{3 i j}=\frac{\partial U_{\gamma}}{\partial x_{i}} \frac{\partial U_{\gamma}}{\partial x_{j}}, \\
c_{\mu}=\frac{c_{\mu 0}\left(1+c_{n S} S^{2}+c_{n \Omega} \Omega^{2}\right)}{1+c_{d S} S^{2}+c_{d \Omega} \Omega^{2}+c_{d S \Omega} S \Omega+c_{d S 1} S^{4}+c_{d \Omega 1} \Omega^{4}+c_{d S \Omega 1} S^{2} \Omega^{2}}, \\
c_{\beta}=c_{\beta 0} \frac{1}{1+m_{d S} S^{2}+m_{d \Omega} \Omega^{2}} .
\end{gathered}
$$

The values of the model constants are $c_{\mu 0}=0.09, c_{n S}=0.005$, $c_{n \Omega}=0.0068, c_{d S}=0.008, c_{d \Omega}=0.004, c_{d S \Omega}=-0.003, c_{d S 1}=$ $0.00005, c_{d \Omega 1}=0.00005, c_{d \Omega \Omega 1}=0.00025, c_{10}=0.40, c_{20}=0.0$, $c_{30}=-0.13, m_{d S}=0.01, m_{d \Omega}=0.003, \sigma_{k}=1.0, \sigma_{\varepsilon}=1.3, c_{\varepsilon 1}=$ 1.44 , and $c_{\varepsilon 2}=1.92$. In the standard $k-\varepsilon$ model, $c_{\mu}=0.09$ and $c_{\beta}=0.0$.

2.2. Large Eddy Simulation. The filtered equations were developed from the Navier-Stokes equations. Consider that $U_{i}$ is a velocity in $i$ direction that contains resolved scale part $\overline{U_{i}}$ and subgrid-scale part $u_{i}$; then $U_{i}=\overline{U_{i}}+u_{i}$; similarly for pressure $P=\bar{p}+p^{\prime}$. Substituting these two decompositions into Navier-Stokes equations, the filtered equations of LES can be obtained as follows.

Continuity equation:

$$
\frac{\partial \overline{U_{i}}}{\partial x_{i}}=0
$$

Momentum equation:

$$
\frac{\partial \overline{U_{i}}}{\partial t}+\frac{\partial \overline{U_{j} U_{i}}}{\partial x_{j}}=g_{i}-\frac{1}{\rho} \frac{\partial \bar{P}}{\partial x_{i}}+\nu \frac{\partial^{2} \overline{U_{i}}}{\partial x_{j}^{2}}-\frac{\partial \tau_{i j}}{\partial x_{j}} .
$$

Here, $\tau_{i j}$ is the subgrid scale Reynolds stress defined by

$$
\tau_{i j}=\overline{u_{i} u_{j}}-\overline{u_{i}} \overline{u_{j}} .
$$

The most commonly used subgrid-scale turbulence model is Smagorisky model proposed by Smagorinsky [8]. In this model, the residual stress takes the Boussinesq eddy viscosity form:

$$
\tau_{i j}-\frac{1}{3} \tau_{k k} \delta_{i j}=-2 v_{t} \overline{S_{i j}} .
$$

Here, the resolved strain rate is $\overline{S_{i j}}=(1 / 2)\left(\partial U_{i} / \partial x_{j}+\right.$ $\left.\partial U_{j} / \partial x_{i}\right)$. model:

The eddy viscosity $v_{t}$ is determined by the Smagorisky

$$
v_{t}=\left(C_{s} \Delta\right)^{2} \sqrt{2 \overline{S_{i j} S_{i j}}} .
$$

$\Delta$ is the filter width related to grid size calculated as the geometric average of the grid spacing in three directions, $\Delta=\left(\Delta x_{1} \Delta x_{2} \Delta x_{3}\right)^{1 / 3} \cdot C_{s}$ is the Smagorisky model constant, whose value varies from 0.065 to 0.20 [9]; 0.15 was used in the present simulation.
TABLE 1: Simulation parameters for different cases.

\begin{tabular}{lccccc}
\hline Cases & Domain size & $\begin{array}{c}\text { Grid } \\
\text { numbers } \\
\text { (variable } \\
\text { spacing) }\end{array}$ & $\Delta t$ & $\begin{array}{c}\text { Truncation } \\
\text { error }\end{array}$ & $\begin{array}{c}\text { Maximum } \\
\text { number of } \\
\text { iterations } \\
\text { per time } \\
\text { step }\end{array}$ \\
\hline $\begin{array}{l}\text { Case 1 } \\
\text { (RANS) }\end{array}$ & $12 r_{0} \times 12 r_{0}$ & $100 \times 100$ & 0.01 & 0.000001 & 200 \\
\hline $\begin{array}{l}\text { Case 2 } \\
\text { (RANS) }\end{array}$ & $12 r_{0} \times 12 r_{0} \times$ \\
$1 r_{0}$ & $70 \times 70 \times 10$ & 0.005 & 0.00001 & 200 \\
\hline $\begin{array}{l}\text { Case 3 } \\
(\text { LES })\end{array}$ & $\begin{array}{c}12 r_{0} \times 12 r_{0} \times \\
1 r_{0}\end{array}$ & $\begin{array}{c}140 \times 140 \times \\
15\end{array}$ & 0.001 & 0.000001 & 200 \\
\hline
\end{tabular}

2.3. Computational Schemes and Flow Domain. The basic equations used in LES were three-dimensional, timedependent, filtered Navier stokes equations. In nonlinear $k-\mathcal{E}$ model, the turbulent stress terms were solved using two additional equations for turbulent kinetic energy $(k)$ and its dissipation rate $(\varepsilon)$. The governing equations for mean velocities and turbulent flows were discretized with the finite volume method based on a staggered grid system. For the momentum equation, convective and diffusive fluxes were approximated with quick and central difference schemes, respectively. The hybrid central upwind scheme was used for the $k$ and $\varepsilon$ equations. Time advancement was achieved by AdamBashforth scheme of second-order accuracy, in each equation. The basic equations were discretized as fully explicit forms and solved successively with the time increment in step by step. The pressure field was solved using iterative procedure at each time step. Three cases have been studied. The initial conditions and simulation details are explained as follows, and the simulation parameters are shown in Table 1 .

2.3.1. Case 1: Simulation of Trailing Vortex by Unsteady RANS. For the $q$-vortex, the direct numerical simulation results were reported by Qin [4]. The simulation for case 1 was performed with the same initial condition of Qin. Since the axial velocity of this vortex is not a function of axial distance but of radial distance, 2D numerical grid was used to simulate 3D flow field. The details of the initial flow field are given as follows.

Tangential velocity:

$$
\frac{V_{\theta}}{V_{0}}=\frac{q_{0}}{r}\left(1-e^{-r^{2}}\right) .
$$

Radial velocity:

$$
\frac{V_{r}}{V_{0}}=0
$$

Axial velocity:

$$
\frac{V_{z}}{V_{0}}=\frac{1}{q_{0}}\left(1-e^{-r^{2}}\right) .
$$

Here, $V_{0}$ is the scaling velocity, related to the initial mean tangential flow, defined as

$$
V_{0}=\frac{M_{p} \gamma}{q_{0}\left(1-e^{-r^{2}}\right)} .
$$




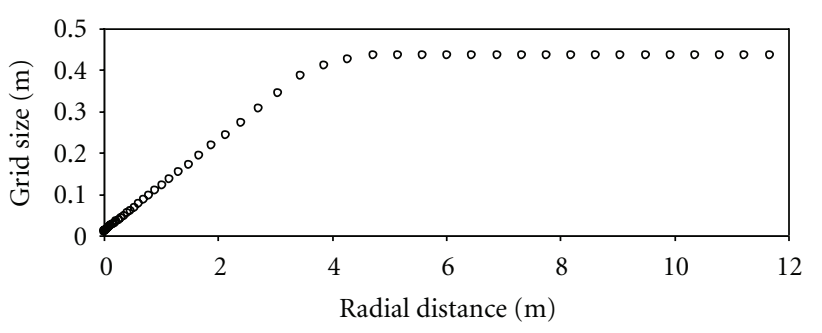

FIGURE 2: Variable grid size with radial distance for case 1.

Here, $M_{p}=0.009$ and $\gamma=1.1209$ were used in Qin's [4] DNS simulation. As reported by Lessen et al. [10], the stability of $q$-vortex can be related to the value of swirl number $q$. In DNS simulation, the initial swirl number $q_{0}$ was chosen to be unity. The time is nondimensionalized by $T=2 \pi r_{0} / V_{\theta m}$ at $t=0$. Here, $r_{0}$ is the radial distance where the tangential velocity contains the peak value in the initial flow field (at $t=0$ ). Variable grid spacing was used with dense grid at centre and coarser towards the boundary. The variable mesh size with radial distance for case 1 is shown in Figure 2. The computational domain consists of 100 grids in each $(x$ and $y$ ) direction. The size of domain was taken sufficiently large $\left(12 r_{0} \times 12 r_{0}\right)$ to overcome the interference of boundary in the vortex decay process. Cartesian grid was used for computation, and the results are presented in cylindrical coordinate using the geometric conversion. The value of $\Delta t$ was taken as $0.01 \mathrm{sec}$. The truncation error was allowed as 0.000001 and the maximum number of iterations per time step was set to be 200 .

2.3.2. Case 2 and Case 3: Simulation of an Ideal Rankine Vortex by Unsteady RANS and LES. These two cases were performed for the same vortex flow field using different simulation methods: case 2 by RANS and case 3 by LES. The initial condition of the vortex is given as follows.

Tangential velocity:

$$
\frac{V_{\theta}}{V_{0}}=-\frac{1}{r}\left(1-e^{-r^{2}}\right) .
$$

Radial velocity:

$$
\frac{V_{r}}{V_{0}}=0
$$

Axial velocity:

$$
\frac{V_{z}}{V_{0}}=0
$$

Here, $V_{0}$ is the scaling velocity, related to the initial mean tangential flow as defined in (16). Like case 1, in these two cases, the initial swirl number $q_{0}$ was also chosen to be unity.

Simulations were performed using 3D Cartesian grid. The size of flow domain for both the cases was $12 r_{0} \times 12 r_{0}$ (in two lateral, $x$ and $y$ directions) $\times 1 r_{0}$ (in axial/depth-wise, $z$ direction). For LES $140 \times 140 \times 15$ grids were used in $x$, $y$, and $z$ directions, respectively. For RANS simulation, the

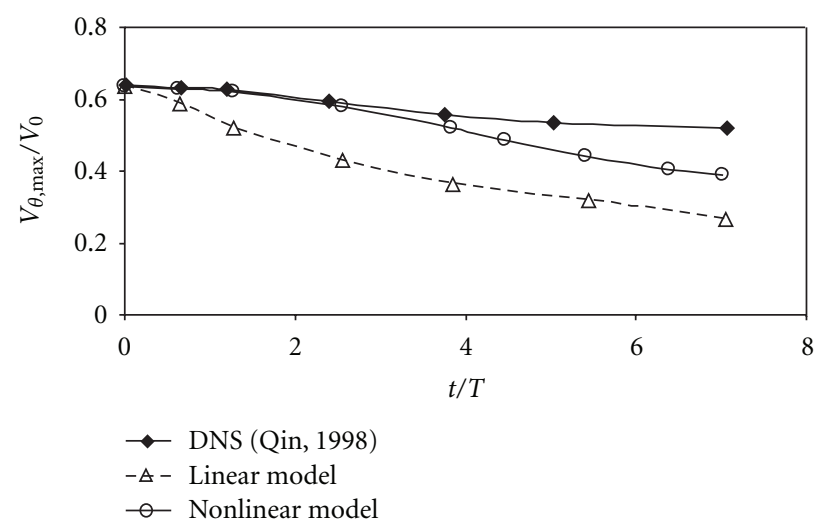

FIgURE 3: Comparison of temporal decay of maximum tangential velocity calculated by RANS with Qin's DNS result.

grid numbers were reduced to $70 \times 70 \times 10$. $3 \mathrm{D}$ staggered variable grid system was used for both RANS and LES. The other parameters for the simulation are shown in Table 1.

\section{Results and Discussions}

At first, the decay of a trailing vortex simulated by standard and non-linear $k-\varepsilon$ model is compared with previous DNS result. Then the decay of an ideal Rankine vortex predicted by nonlinear $k-\varepsilon$ model and large eddy simulation (LES) is compared. The comparative discussion of simulated results is presented here in after.

\subsection{Comparison of RANS Prediction with Qin's DNS Data}

3.1.1. Comparison of Temporal Decay. To investigate the predictability of standard (linear) and non-linear $k-\varepsilon$ model, the decay of trailing vortex as defined in case 1 was simulated with the same initial conditions of Qin [4] using both models. In this section, the simulated results are compared with Qin's DNS data. Comparison of temporal decay of maximum tangential velocity is shown in Figure 3 . It is clearly seen that the standard $k-\varepsilon$ model predicts much faster decay than that of non-linear model. Although the nonlinear model shows better comparison with DNS calculation, the decay rate is still faster than that of DNS prediction, especially at about $t>4.0 T$.

The radial distribution of circumferential velocity, circulation, and axial velocity is compared with the DNS calculation at time $t=3.72 \mathrm{~T}$, as shown in Figures 4, 5 , and 6, respectively. At this age the vortex has already overcome the effect of initial conditions and the turbulent flow field became saturated and stabilized gaining a peak value of the turbulent kinetic energy after passing its growth periods. In these figures, the non-linear model shows much better agreement with DNS data compared to standard model. Even for non-linear model, the magnitude and position of maximum tangential velocity show slightly faster decay than DNS data. Such discrepancy is also seen in the comparison of circulation profile. The cause of deviation 


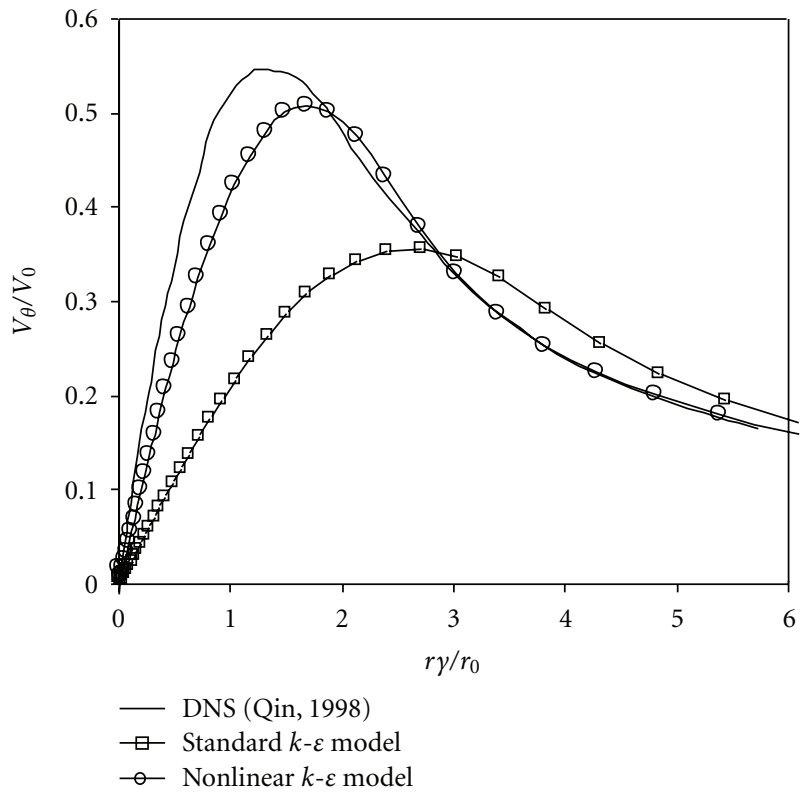

FIGURE 4: $k-\varepsilon$ model prediction of tangential velocity profile compared with Qin's DNS results $(t=3.72 T)$.

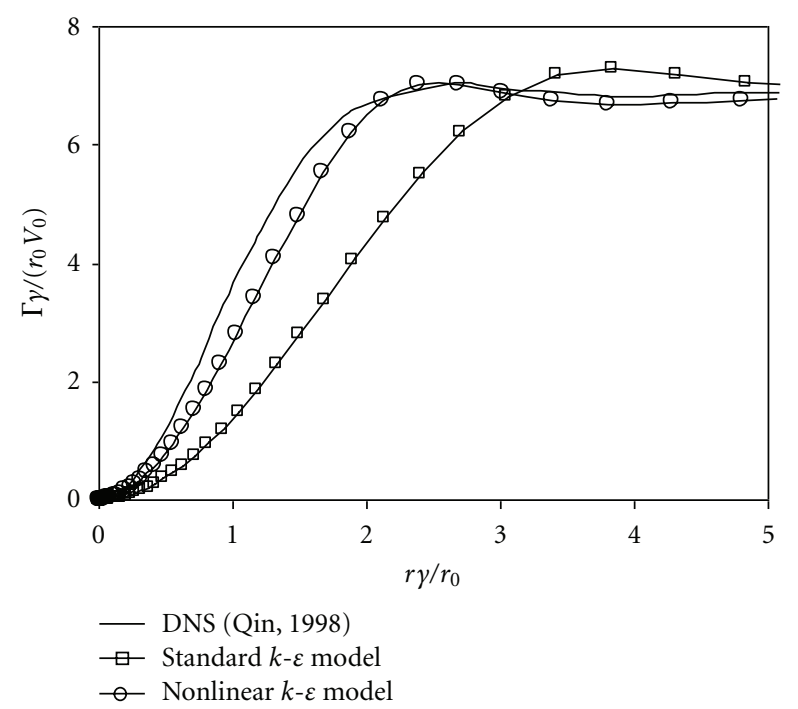

FIGURE 5: $k-\varepsilon$ model prediction of circulation profile compared with Qin's DNS results $(t=3.72 T)$.

is explained by comparing the temporal decay of vortex with the growth/decay of turbulence in the next section.

3.1.2. Temporal Growth/Decay of Turbulence. Production of turbulence in the vortex field is due to the tangential and axial velocity gradients. In the vortex lifetime, five different growth/decay rates of turbulent kinetic energy as reported in DNS calculation [4] were also reflected in the simulation of non-linear $k-\varepsilon$ model. Figure 7 presents the growth/decay of turbulence kinetic energy with approximated five different time periods. Initially (zone (i)), the vortex changes very slowly, as it requires adjustment of any unphysical nature of

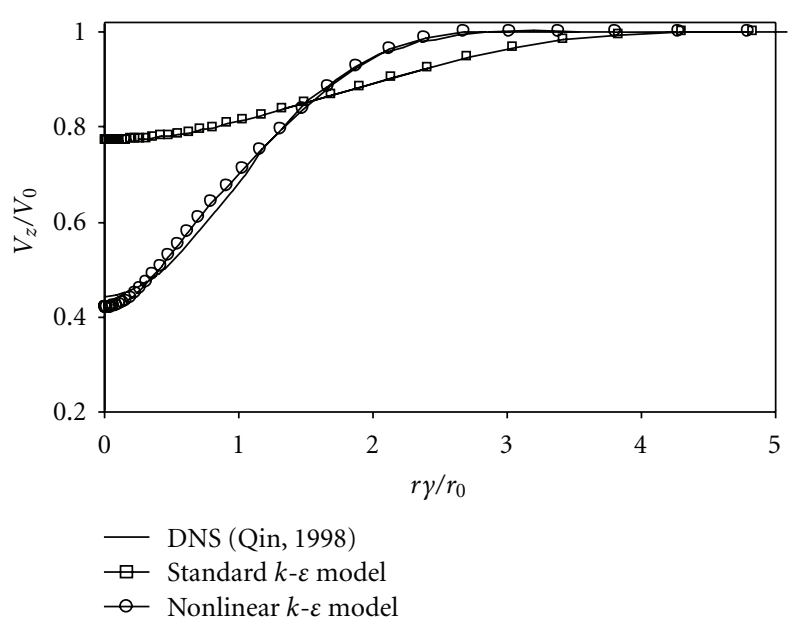

FIGURE 6: $k-\varepsilon$ model prediction of axial velocity profile compared with Qin's DNS results $(t=3.72 T)$.

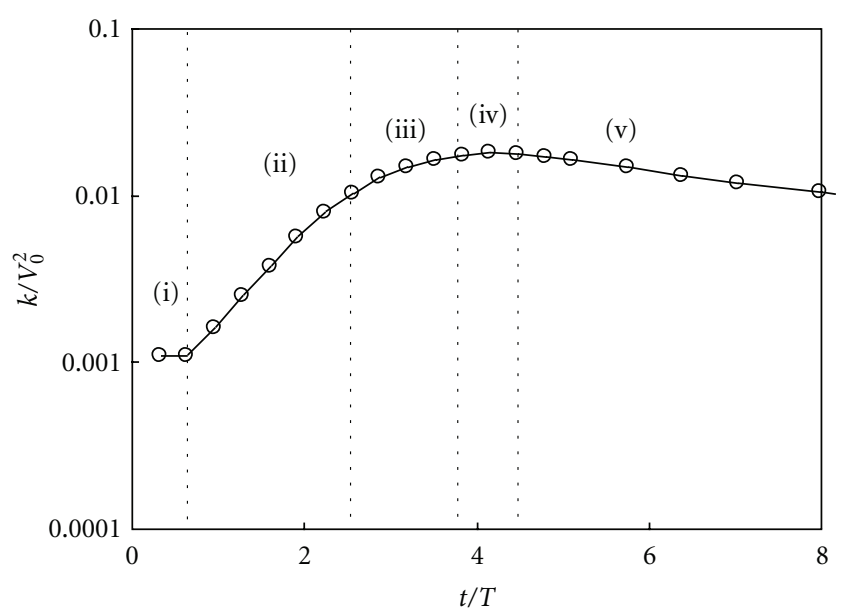

FIGURE 7: Growth/decay of turbulent kinetic energy with different time periods (nonlinear $k-\varepsilon$ model).

initial conditions. In time zone (ii), an exponential growth of turbulent kinetic energy is seen. The growth slows down in the next time period of zone (iii). It is found that the growth of axial velocity is significantly higher in time zone (ii) and (iii). The turbulent kinetic energy reaches its peak value and remains almost constant throughout the short period of zone (iv). It reveals that in this stabilization period the flow field becomes saturated and cannot support additional turbulence. Finally, the decay of turbulence is started as predicted by most of the previous researches (such as $[2,11]$, etc.). The logarithmic plot depicts that the decay rate in zone (v) is much slower than the growth rate in zone (ii). The decay of velocity field slows down as the turbulence decay period starts.

Comparing Figure 3 with Figure 7, we can conclude that in the growth phase as well as in the stabilized phase of turbulence, the decay rate of tangential velocity by nonlinear $k-\varepsilon$ model is well comparable with DNS result. However, in the decay phase of turbulence, RANS model shows faster 


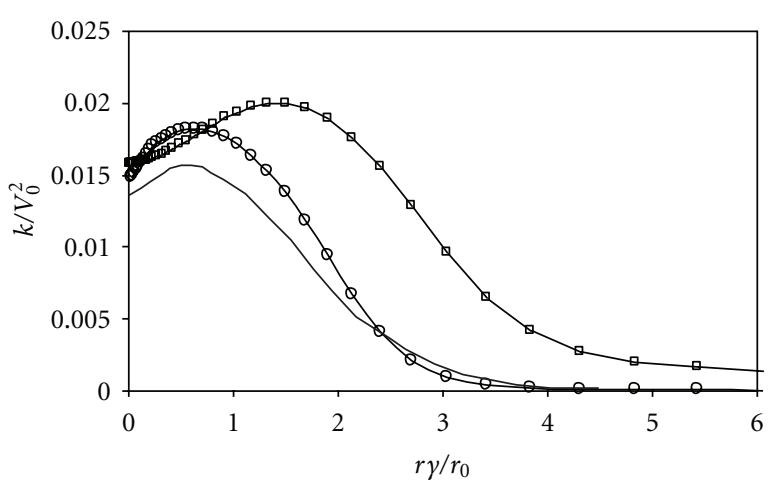

- DNS (Qin, 1998)
$\square-$ Standard $k-\varepsilon$ model
$\square-$ Nonlinear $k-\varepsilon$ mode

FIGURE 8: $k-\varepsilon$ model prediction of turbulent kinetic energy compared with Qin's DNS results $(t=3.72 T)$.

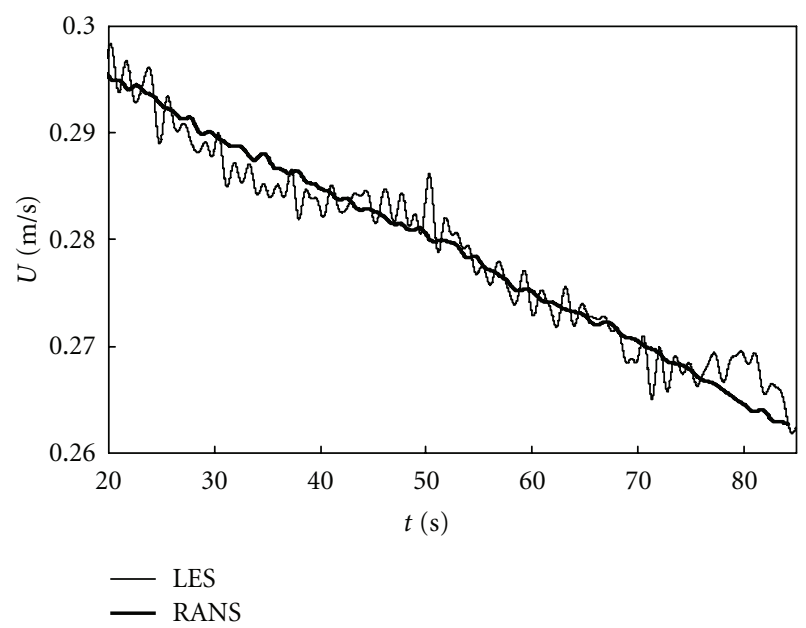

FIgURE 9: Comparison of temporal fluctuations of velocity at a point by RANS and LES simulations.

decay of tangential velocity due to its slower decay of turbulence compared to DNS.

Figure 8 depicts the comparison of the radial distribution of turbulent kinetic energy using three models. It is seen from the figure that the $k-\varepsilon$ model overpredicts the kinetic energy at the peak compared to DNS. This is the cause for the faster decay of tangential velocity in the $k-\varepsilon$ model (as observed in Figure 4).

3.2. Decay of an Ideal Rankine Vortex by LES and RANS Simulations. In this $3 \mathrm{D}$ simulation, the vortex flow field was given to rotate with the vertical axis in a free surface rectan gular domain. The temporal change of velocity at a point is shown in Figure 9 for both LES and RANS simulations. In addition to temporal decay of velocity, the typical velocity fluctuations are observed in LES results; on the other hand, RANS simulation shows a mean decay profile.

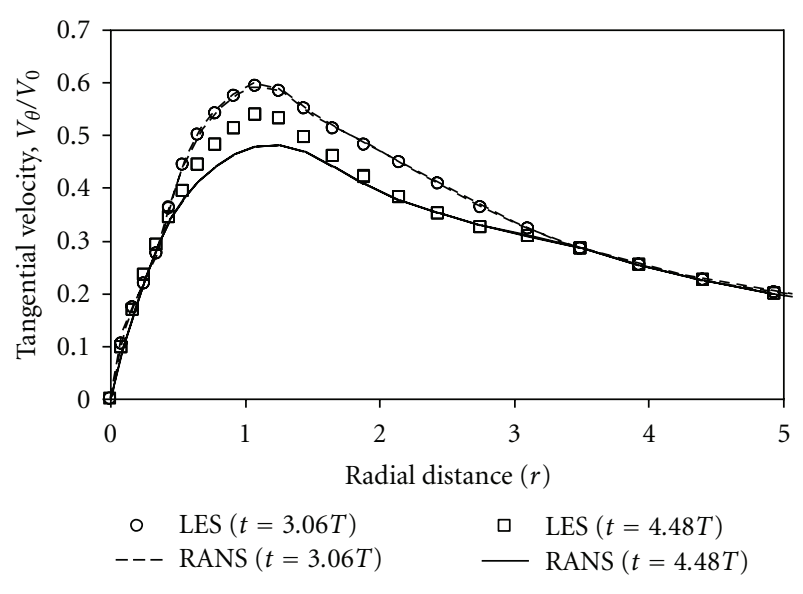

Figure 10: Radial distribution of depth calculated by RANS and LES (symbols indicates LES prediction, and lines show the corresponding RANS prediction).

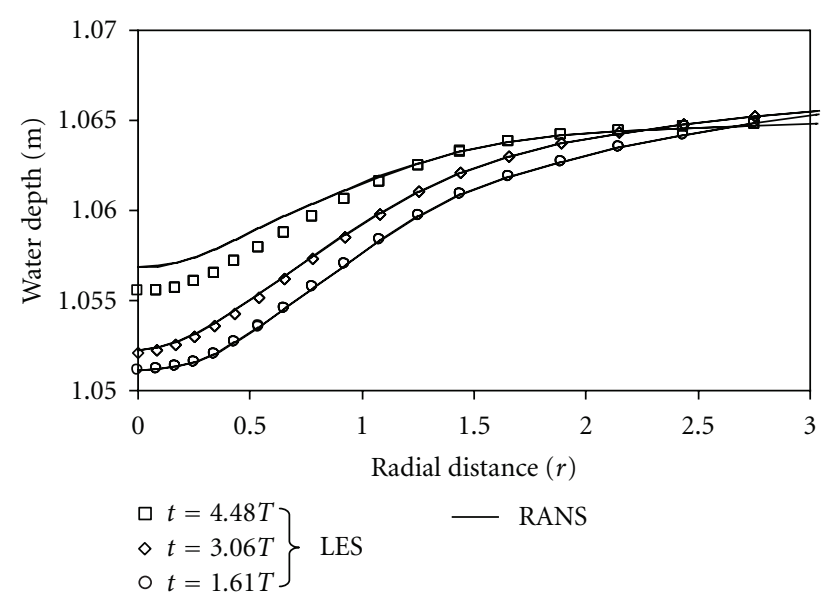

FIGURE 11: Radial distribution of tangential velocity calculated by RANS and LES.

3.2.1. Comparison of Temporal Decay. Figure 10 shows the radial distribution of tangential velocity predicted by LES and RANS models at $t=3.06 \mathrm{~T}$ and $t=4.48 \mathrm{~T}$. Although the velocity profiles at $t=3.06 T$ show good comparison, RANS model shows faster decay than LES at $t=4.48 T$.

The variation of water surface for different times predicted by RANS and LES is shown in Figure 11. The water surface is found to be depressed near the central area with minimum water depth at the centre of vortex. Since the strength of vortex decreases due to the decay of tangential velocity, the amount of depression in water surface is also de creased with time. Similar to Figure 10, although the depression of water surface at $t=3.06 \mathrm{~T}$ shows good comparison, RANS model predicted less depression in water surface than that of LES at $t=4.48 T$.

Figure 12 shows the calculated temporal decay of maxi mum tangential velocity for both LES and non-linear $k-\varepsilon$ model. It is found that although the prediction of RANS model shows good agreement with LES in initial times, some 


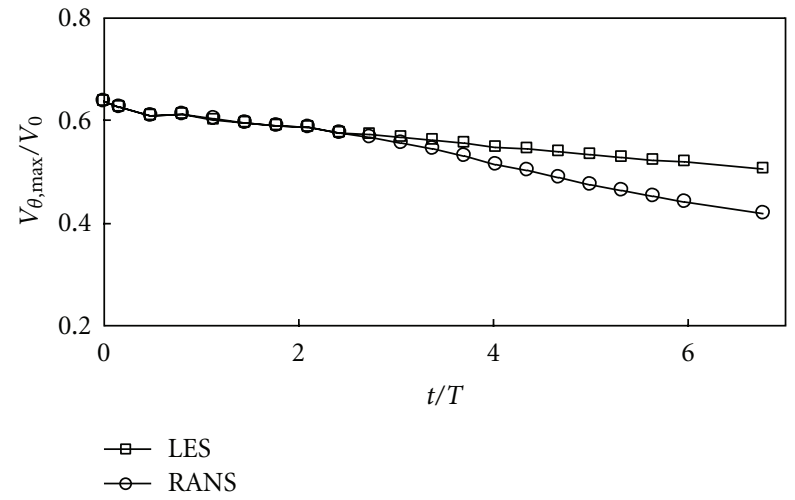

Figure 12: Time decay of maximum tangential velocity calculated by RANS and LES.

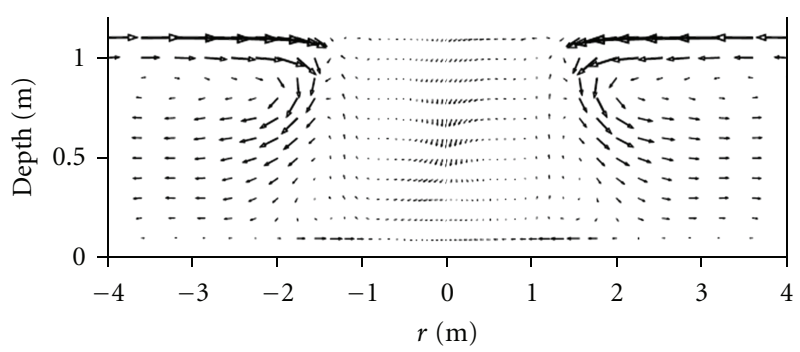

(a)

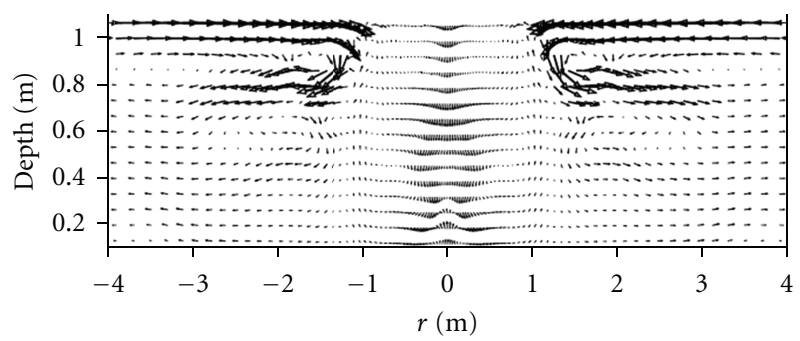

(b)

FIGURE 13: Pattern of secondary current at $t=40 \mathrm{sec}$ by (a) RANS and (b) LES simulations.

deviation is observed at about $t>4.0 T$. Such discrepancy in the decay phase of turbulence is also observed in the comparison of non-linear $k-\varepsilon$ model predictions with previous DNS results of trailing vortex decay (case 1). Therefore, similar to previous case, it can be concluded that in the growth phase as well as in stabilized phase of turbulence, the decay rate of tangential velocity by RANS model is well comparable with LES. However, in the decay phase of turbulence, RANS model shows slightly faster decay of tangential velocity due to its slower decay of turbulence compared to LES.

3.2.2. Secondary Currents. The secondary currents calculated by both the models are shown in Figure 13. The flow vectors of secondary current show three circulation cells in each side of the centerline. Secondary current inside the vortex core is found relatively weaker than the outside of core. It is ex- plained that (in Figure 1) the Rankine vortex is a combination of nearly solid body rotation and potential vortex. For both type of vortices, the individual patterns of secondary currents are reported by Laugt [12]. The pattern of secondary currents predicted by LES as well as RANS is found well agreed to Laugt. In the vortex core, the secondary current shows the pattern similar to that of solid body rotation; outside the core, the pattern is similar to that of potential vortex. The patterns as well as magnitudes of secondary currents predicted by two models are well comparable to each other.

\section{Conclusions}

3D numerical simulations were carried out for an ideal Rankine vortex using both RANS (non-linear $k-\varepsilon$ ) and LES models. In the growth phase as well as in stabilized phase of turbulence, the decay rate of tangential velocity by RANS model was found to be well comparable with LES. However, in the decay phase of turbulence, RANS model showed faster decay of tangential velocity compared to LES due to its slower decay of turbulence. The patterns as well as magnitudes of secondary currents predicted by two models are in good agreement with each other. The water surface was found to be depressed near the central area of the vortex with a minimum water depth observed at the centre of vortex.

In order to investigate the predictability of standard (linear) and non-linear $k-\varepsilon$ models, the decay of a trailing vortex was simulated and compared with previous DNS data. The comparison of non-linear $k-\varepsilon$ model prediction with previous DNS result showed similar phenomenon as observed in the comparison with LES as described previously. Due to the inherent deficiency of standard $k-\varepsilon$ model, it predicted much faster decay than that of non-linear model.

\section{References}

[1] W. R. C. Phillips, "The turbulent trailing vortex during rollup," Journal of Fluid Mechanics, vol. 105, pp. 451-467, 1981.

[2] M. S. Uberoi, "Mechanisms of decay of laminar and turbulent vortices," The Journal of Fluid Mechanics, vol. 90, no. 2, pp. 241-255, 1979.

[3] P. G. Saffman, "The structure and decay of turbulent trailing vortices," Archives of Mechanics, vol. 26, no. 3, pp. 423-439, 1974.

[4] J. H. Qin, Numerical simulation of a turbulent axial vortex, Ph.D. thesis, Purdue University, West Lafayette, Ind, USA, 1998.

[5] I. Kimura, W. S. J. Uijttewaal, T. Hosoda, and M. S. Ali, "URANS computations of shallow grid turbulence," Journal of Hydraulic Engineering, vol. 135, no. 2, pp. 118-131, 2009.

[6] M. S. Ali, T. Hosoda, and I. Kimura, "Unsteady simulation of turbulent axial vortex using non-linear $k-\varepsilon$ model," Journal of Applied Mechanics, vol. 11, pp. 821-832, 2008.

[7] I. Kimura and T. Hosoda, "A non-linear k- $\varepsilon$ model with realizability for prediction of flows around bluff bodies," International Journal for Numerical Methods in Fluids, vol. 42, no. 8, pp. 813-837, 2003.

[8] J. Smagorisky, "General circulation experiments with primitive equations-I: the basic experiment," Monthly Weather Review, vol. 91, pp. 99-165, 1963. 
[9] J. H. Ferziger and M. Peric, Computational Methods for Fluid Dynamics, Springer publications, New York, NY, USA, 2nd edition, 1999.

[10] M. Lessen, P. J. Singh, and F. Paillet, "The stability of a trailing line vortex. Part I: inviscid theory," Journal of Fluid Mechanics, vol. 63, no. 4, pp. 753-763, 1974.

[11] G. K. Bachelor, "Axial flow in trailing line vortices," The Journal of Fluid Mechanics, vol. 20, no. 4, pp. 645-658, 1964.

[12] H. J. Laugt, Vortex Flow in Nature and Technology, John Wiley \& Sons, New York, NY, USA, 1983. 

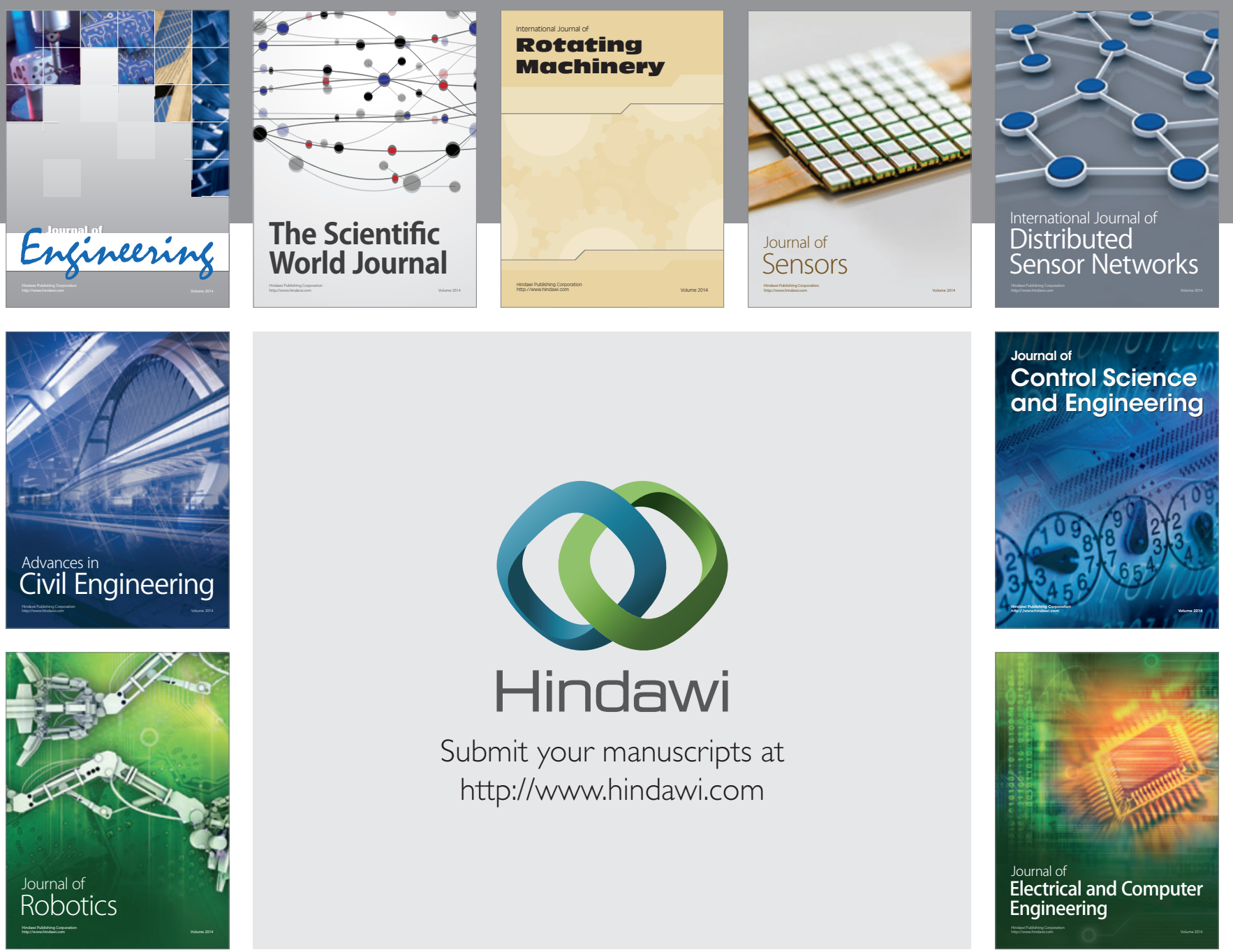

Submit your manuscripts at

http://www.hindawi.com
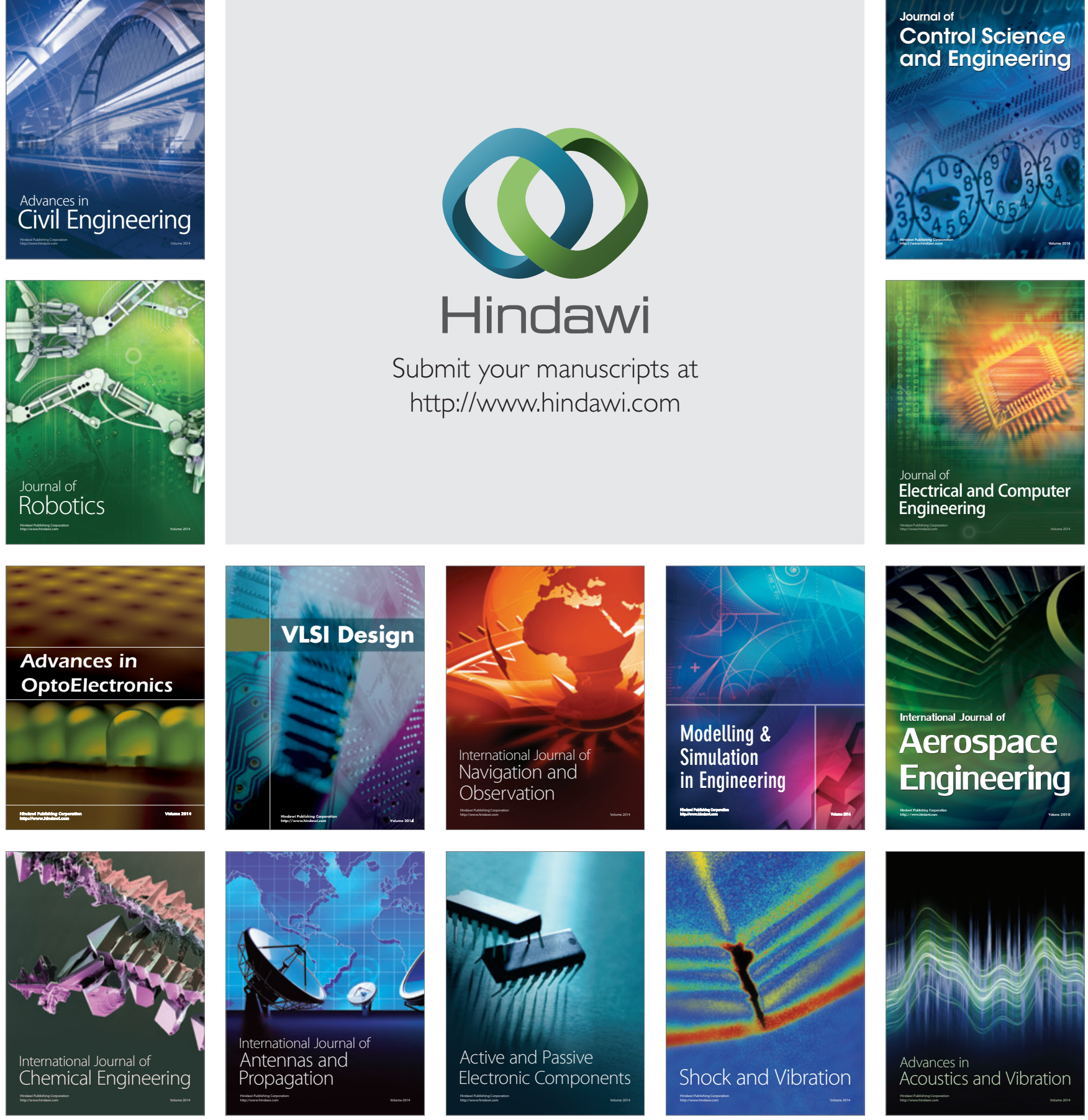